\title{
Knots in the Nursery: (Cats) Cradle Song of James Clerk Maxwell
}

\author{
Daniel S. Silver
}

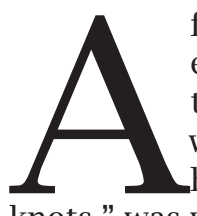

field of mathematics can acquire energy from a notable paper. I will wager that knot theory is the only field that was both propelled by a paper and heralded by a poem. The paper, "On knots," was written by the Scottish mathematician and physicist Peter Guthrie Tait in 1877 . The poem, (Cats) Cradle Song, was a witty response by James Clerk Maxwell, another Scottish mathematician as well as a physicist, who today ranks alongside Newton and Einstein.

Many associate Maxwell only with the eponymous equations that relate electricity and magnetism. Sadly, scientists are seldom remembered for their general intellect or sense of humor. ${ }^{1}$ Maxwell's writing reveals an abundance of both.

Tracing Maxwell's literary influences is trickier than chasing down his scientific ones. Maxwell's biographer, Lewis Campbell, boasts of his subject's wide-ranging tastes in literature: "[Maxwell's] acquaintance not only with scientific literature, but with nearly every other class of books was astonishing." Lewis Carroll was among Maxwell's favorite authors. ${ }^{2}$

Maxwell's enjoyment of books was matched by his obsession with puns. They were more than exercises for his sprinting mind. In a Cambridge essay of 1858, Maxwell explained:

Daniel S. Silver is professor of mathematics and statistics at the University of South Alabama. His email address is silver@southa1abama.edu.

${ }^{1}$ Einstein is the premier exception.

${ }^{2}$ Writing backwards, Maxwell asked Tait in 1873, "Why have you forgotten to send Alice? We remain in Wonderland till she appears." Later he sends another note: " $\Theta \alpha \gamma \xi \quad \phi о \rho$

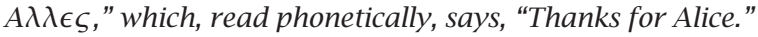
DOI: http://dx.doi.org/10.1090/noti1171
Now, as in a pun two truths lie hid under one expression, so in an analogy one truth is discovered under two expressions. Every question concerning analogies is therefore the reciprocal of a question concerning puns, and the solutions can be transposed by reciprocation.

Evidence of Maxwell's humor leaps from the many letters and postcards he wrote to Tait, his lifelong friend. It is found also in poems that he wrote for the amusement of his colleagues. Sometimes biting, always witty and enigmatic, the poems mirrored the personality of their author.

(Cats) Cradle Song was composed just before Maxwell was diagnosed with a disease that would take his life at the age of forty-eight. Unlike $A$ Paradoxical Ode, Maxwell's dark, last poem, (Cats) Cradle Song is sunny and lighthearted. Its purpose was to have a bit of good-natured fun at Tait's expense.

Our purpose is to have some fun exploring the lines of (Cats) Cradle Song. We will try to capture some of the playful spirit that brought knot theory into being. References to mathematical ideas that were novel at the time will emerge. Some of the ideas would endure and inspire succeeding generations.

\section{A Knotty Backstory}

Tait thought of a knot just as mathematicians do today, as a closed loop in ordinary 3-dimensional space. Similarly, a link is a collection of knots, no two intersecting. One should think of loops of highly elastic material. Two knots or links are thought to be the same if one can be deformed into the other without breaking. Showing that two knots 


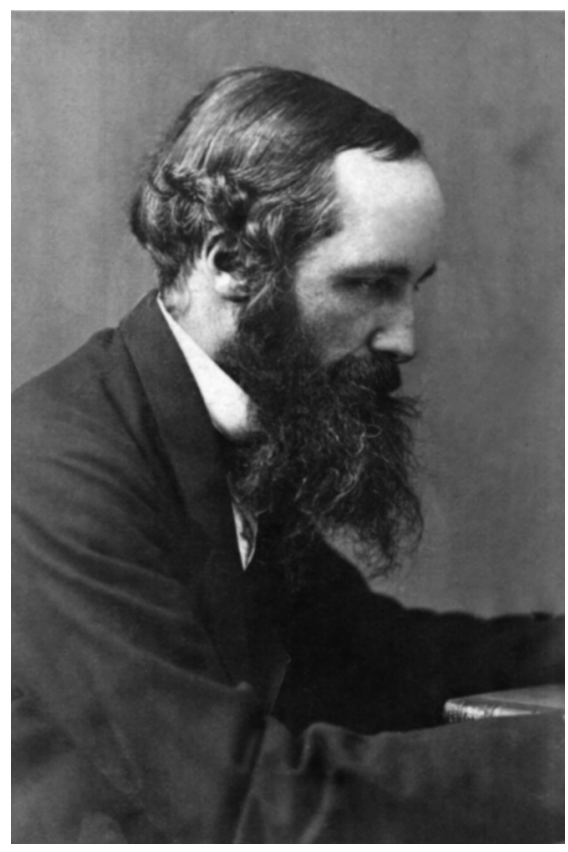

James Clerk Maxwell

or links are different can be a formidable task, but one that can be surmounted with ideas from algebra, combinatorics, or hyperbolic geometry. The past three decades have seen an abundance of new knottheoretic tools, some of them having relationships with methods of mathematical physics. Today, knot theory is one of the most active areas of topology.

Knots have bound the attention of mathematicians for more than two centuries. While Carl Friedrich Gauss considered knots mathematically, the first sustained study of them began with Tait about the year 1876. And it began oddly.

Tait's enthusiasm for knots was sparked by Hermann von Helmholtz. The German physician and physicist had shown that knots of ether, a subtle, frictionless fluid thought to permeate the universe, would persist eternally. In Tait's language, knots of ether "will for ever remain stamped with that vortex-motion at least until the creative act which produced it will take it away again."

Tait had many enthusiasms. Pipe smoking was another. In his mind, smoke rings were a crude but instructive model of etherial vortices. He fashioned a pair of devices to manufacture smoky rings and demonstrated them for his colleague William Thomson, later Lord Kelvin. What he made were two simple wooden boxes, each with a large opening in the back and a small hole in front. A piece of India rubber was stretched over the backs. Inside the boxes Tait placed dishes of common salt and sulphuric acid. When he struck the back of each box, rings of hydrogen chloride gas emerged.

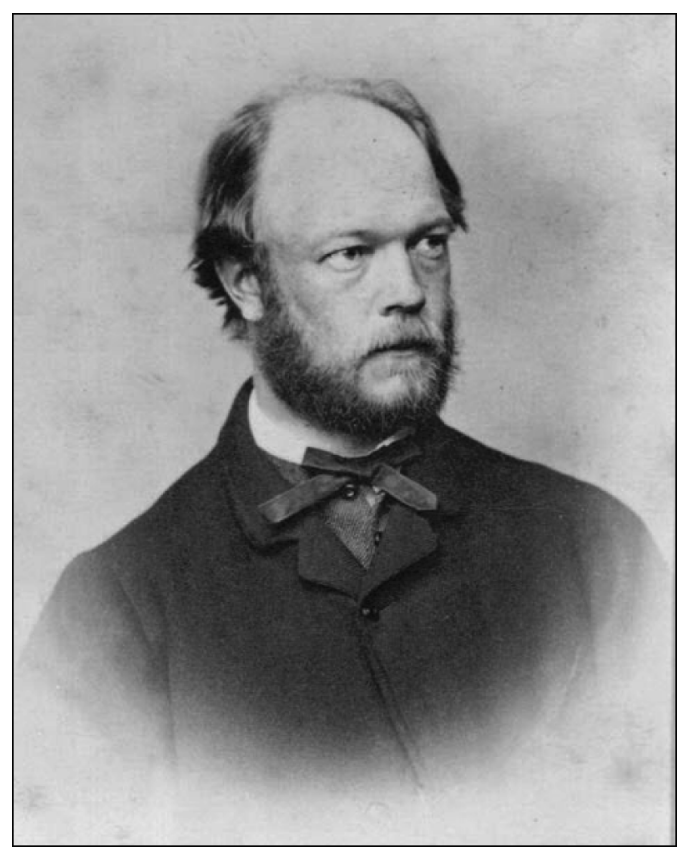

Peter Guthrie Tait

As the reagents combined, so too did Thomson's thoughts. Observing the poisonous rings perform their silent acrobatics, Thomson concluded that chemical elements must in fact be knotted vortices of ether. Different knots then must constitute different elements. So was born the vortex atom theory.

But the theory was nearly stillborn. In June 1868, French mathematician Joseph Bertrand announced in the journal Comptes Rendus what he believed to be a major flaw in Helmholtz's article. Tait seemed ready to abandon the vortex atom theory. To Thomson he wrote:

Did you see the Comptes Rendus? In that for the 22nd June (I think) Bertrand states that there is a mistake in the beginning of $\mathrm{H}^{2}$ 's Vortex paper ${ }^{3}$ which renders ALL HIS RESULTS ERRONEOUS. So, of course, you may drop your vortex-atom-paper, and come home to useful work. B. does not point out the mistake, nor have I been able to find it-but $\mathrm{H}^{2}$ will perhaps be able to tell you.

The analogy between fluid flow and electromagnetism had already persuaded Maxwell to follow closely the developments in the vortex atom theory. Aware of his interest, Thomson suggested that Tait

\footnotetext{
${ }^{3}$ Experimental initialism such as this was popular among British scientists of the nineteenth century. In his book Flatland (1884), the author Edwin Abbott Abbott referred to himself as "A Square." Maxwell often replaced his initials with $\frac{\partial p}{\partial t}$, short for the equation JCM $=\frac{\partial p}{\partial t}$, expressing a law of thermodynamics.
} 


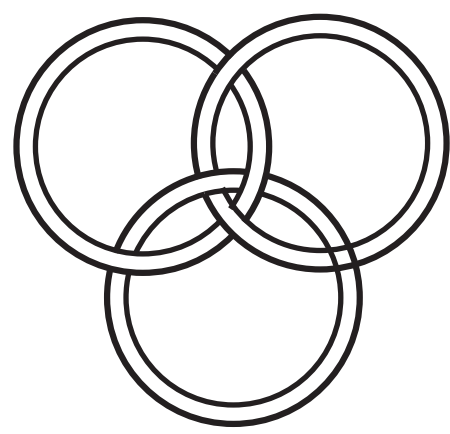

Figure 1. Borromean rings from Tait's paper "On knots."

ask for Maxwell's opinion of Bertrand's criticism. A fortnight brought reassurance from Maxwell:

I do not see the Comptes Rendus, nor do I perceive, without the aid of Bertrand, the 'légère faute' in $\mathrm{H}^{2}$. In fact I consider it impossible to commit one at the beginning of such a theory. You must either tell a 'rousing whid'4 or be infallible.

Maxwell added that he had checked the equations in Helmholtz's paper and found them to be correct. If Maxwell is responsible for extending the life of the vortex atom theory, then he should receive credit also for Tait's eventual plan to develop a mathematical theory of knots.

Nobody knows why a decade passed before Tait began his project of knot classification. Nevertheless, by 1877 he was completely immersed in it. After reading several short papers about knots to the Royal Society of Edinburgh, Tait began to organize his results for a large work. "On knots" would be the first mathematics publication with the word "knots" in its title.

Until now Tait had been unaware of work done by Johann Benedict Listing, a German mathematician and former student of Gauss. It was Listing who coined the word "topology." He had used it in the title of a publication, "Vorstudien zur Topologie" (1847). A later paper by Listing, "Der Census räumlicher Complexe," contains first steps in the direction of homology theory, steps that Maxwell embraced in his Treatise on Electricity and Magnetism (1873). In an amusing postcard dated January 22, 1877, Maxwell informed Tait that, if he was looking for Listing's work on knots, then he was reading the wrong paper:

You are reading a paper in $4^{\circ}$ in Gött. Comm.

["Der Census räumlicher Complexe"]. The

${ }^{4}$ The phrase "rousing whid" is from the poem Death and the Doctor by Scottish poet Robert Burns. Tait would have appreciated the reference to the work of a fellow countryman. knots are in an older paper ["Vorstudien zur Topologie"].... There is good stuff in the $4^{\circ}$ paper but knot nots.

Tait, aware of Maxwell's mastery of the literature, asked his friend for comments about a draft of "On knots." On June 30, Tait wrote to Maxwell:

Would it bother you if I sent you the M.S.S. of my paper Knots prepared for Trans R.S.E.? If not I wish you would look it over. I have got so thoroughly on one groove that I fear I may be missing or unduly exalting something which will appear excessively simple to any one but myself. You are just the party to detect this.

In the letter, Tait confessed that he was baffled about questions of linking:

Here for instance, is one of my difficulties. What are we to call the "belinkedness" of the arrangement [Figure 1], where there is no linking at all \& yet you can't separate the rings? If you change any one sign [crossing] a ring comes off, but one degree of linkedness is introduced! This is neither Knot nor Link. What is it?

The link that Tait described had once been favored by the sculptor Michelangelo, who used it (with the letter "M") to mark his stones. Its current name, "Borromean rings," derives from its heraldic use by the Borromeo family of northern Italy.

In his letter, Tait continued:

The M.S.S. is with the printer, and I could have moved the Council R.S.E. to inflict it on you-but I don't like to bother you unless you are pleased to be bothered. Neill says his type is at present all locked ${ }^{6}$ by Plarr's \& Milne Home's papers so that you can have the M.S.S. for 3 weeks if you like.

Whether Tait received any helpful comments from Maxwell is not clear. At the least, he got a poem. (Cats) Cradle Song appears in biographies both of Maxwell and Tait.

\footnotetext{
${ }^{5}$ Here is typical Maxwellian humor. Not satisfied with the simple pun "not knots," Maxwell has interchanged the roles of the two words.

${ }^{6}$ In this precomputer age, publications were set up in lead type.
} 
(Cats) Cradle Song, By a Babe in Knots.

PETER the Repeater, Platted round a platter Slips of slivered ${ }^{7}$ paper, Basting them with batter.

Flype 'em, slit 'em, twist 'em, Lop-looped laps of paper;

Setting out the system

By the bones of Neper.

Clear your coil of kinkings Into perfect plaiting,

Locking loops and linkings Interpenetrating.

Why should a man benighted, Beduped, befooled, besotted, Call knotful knittings plighted, Not knotty but beknotted?

It's monstrous, horrid, shocking, Beyond the power of thinking,

Not to know, interlocking Is no mere form of linking.

But little Jacky Horner

Will teach you what is proper, So pitch him, in his corner, Your silver and your copper.

\section{Untangling (Cats) Cradle Song}

Cat's-cradle, a children's string game known around the world, had come to England recently. ${ }^{8}$ It is mentioned in Anthony Trollope's The Last Chronicle of Barset, a popular novel first published in 1867 and conceivably one that Maxwell had read. Maxwell, the restless punster, separates "Cats" in parentheses and emphasizes "Cradle Song." His poem is a friendly tease of a grown man playing with knots. Maxwell, a playmate, signs himself "a Babe in Knots." Possibly, "a Babe in Knots" is a wordplay on "babe in arms." However, it might also be Maxwell's variation of "Babes in the Wood," the name of a traditional story about children lost in a dangerous forest. ${ }^{9}$

\footnotetext{
${ }^{7}$ In some publications the word "silvered" appears in place of "slivered." The version here agrees with that found in The Life of James Clerk Maxwell.

${ }^{8}$ Writing about cat's-cradle in 1906, ethnologist Alfred Hadden concluded that it must have been introduced into England "within relatively recent years as there are no references to it in the older literature."

${ }^{9}$ In 1879, watercolorist Randolph Caldecott illustrated the story for George Routledge and Sons, London. Maxwell's
}

\section{Peter the Repeater}

Peter the Repeater is, of course, Peter Guthrie Tait, "repeater" being one who recites a lesson. Maxwell might have been alluding to the popular English nursery rhyme about "Peter Pumpkin Eater."10

\section{Flype 'em, Slit 'em, Twist 'em}

In the poem, Tait is "platting" or braiding strips of paper, twisting, gluing and slitting them. "On knots" is more explicit: "Give the slip one half twist (i.e., through $180^{\circ}$ ) before pasting the ends together. The edges now form one continuous curve." What Tait described would later be called a Möbius band, named after August Ferdinand Möbius, one of two mathematicians who discovered it simultaneously in 1858. The other mathematician was Listing. His drawing, found in "Der Census räumlicher Complexe", was the first published image of the band.

Tait explored variations. For example, on page 297 of "On knots," he wrote: "Give one full twist before pasting. Each edge forms a closed curve. The curves are, in fact, once linked with each other." He continued: "Now suppose that, in any of the above examples, after the pasting, we cut the slip of paper up the middle throughout its whole length." The Möbius band remains connected while the band with a full twist becomes "two interlinking single coils, each having one whole twist."

When a band with three half twists is cut up the middle, the result is "a trefoil knot, and when flattened it has three whole twists." This and the other variations, using any number of halftwists, were explained in "Der Census räumlicher Complexe", yet Tait failed to acknowledge it in his paper. In Listing's obituary, which Tait wrote for Nature in 1883, grudging amends are made. After comparing Listing's examples to "a well-known juggler's ${ }^{11}$ trick," Tait added:

This remark of Listing's forms the sole basis of a work which recently had a large sale in

Vienna: - showing how, in emulation of the

favorite cousin Jemima Wedderburn Blackburn was another famous watercolorist of the period. I conjecture that Maxwell knew Caldecott and had his forthcoming book in mind.

${ }^{10}$ Just a few years earlier, in 1868, James Riddell published a collection of verse, Aberdeen and Its Folk. In it one finds an early, disturbing version:

Peter, my neeper

Had a wife,

And he couidna' keep her.

He pat her i' the wa',

And lat a' the mice eat her;

Maxwell would have had interest, since he held his first professorship at the University of Aberdeen.

${ }^{11}$ In nineteenth century Britain, the word "juggler" meant someone who entertains people, often with tricks. 


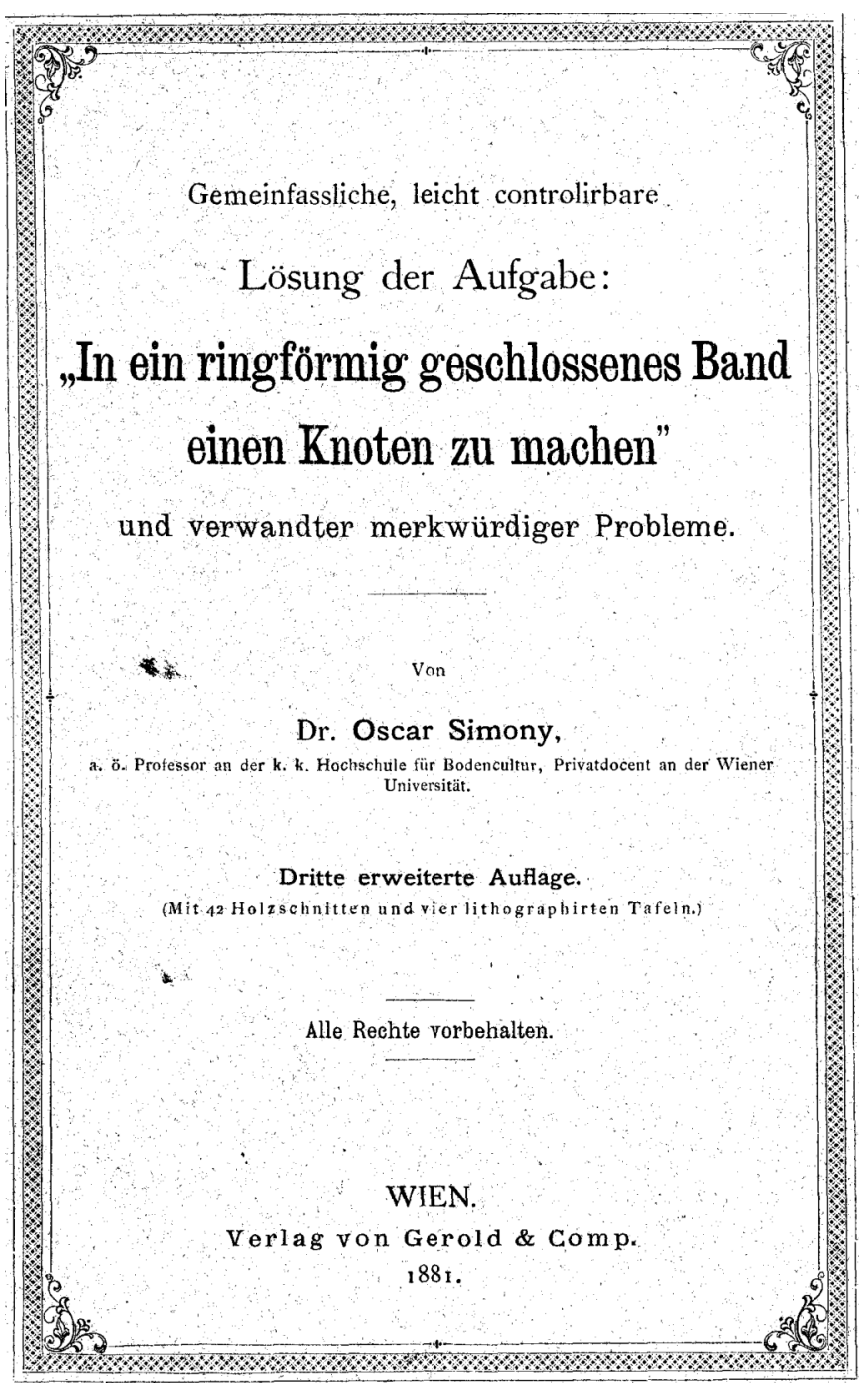

Figure 2. Title page of Simony's publication, 3rd edition, Vienna.

celebrated [Henry] Slade, to tie an irreducible knot on an endless string!

Henry Slade was a famous American psychic, believed by many to have paranormal abilities. Slade had given seances in England in the summer of 1876. When his deceptions were discovered, he was tried, convicted of fraud, and sentenced to three months of hard labor. Slade managed to avoid prison by a legal technicality, and he proceeded to Germany and Austria, where Spiritualism was growing in popularity.

Johann Zöllner, a professor of physics and astronomy at the University of Leipzig, had unwavering faith in Slade. He believed that spirits resided in four-dimensional space. That any knot can be undone in a such a space had become known. ${ }^{12}$ Zöllner was convinced that Slade was

\footnotetext{
${ }^{12}$ In "On knots" Tait gives a reference to a paper of Klein (Mathematische Annalen, ix. 478) in which the result is not specifically mentioned. Klein, realizing that he could prove
}

tying knots in endless strings with the aid of an extra dimension. The work that Tait reported had sold so well in Vienna was an illustrated pamphlet by a friend of Zöllner, the mathematician Oscar Simony (see Figure 2).

Tait was always the scientist. In an article in Contemporary Review, "On the teaching of natural philosophy," he declared: "There is nothing so well fitted like mathematics to take the nonsense out of a man." Still, Tait's views on the possibility of a spatial fourth dimension must have been complicated. He had recently published a bizarre book, The Unseen Universe, with co-author Balfour Stewart, in which he proposed that we live alongside infinitely many parallel universes of material approximating perfect ether. In these universes, Tait believed, thoughts and souls might dwell.

Maxwell, ever cautious about speculation, especially on matters of faith, was more doubtful about the possibility of a spatial fourth dimension. In a letter to a friend in 1871, he wrote:

The peculiarity of our space is that of its three dimensions none is before or after another. As is $\mathrm{x}$, so is $\mathrm{y}$ and so is $\mathrm{z}$.

If you have 4 dimensions this becomes a puzzle, for first, if three of them are in our space, then which three?

\section{Setting Out the System by the Bones of Neper}

The main goal of "On knots" was enumeration. Tait would first have to find a way to encode knots.

Since ancient times, knots and links have been depicted by diagrams on flat surfaces, projections with the generic property that at most two segments cross anywhere and do so transversely. The artistic "hidden line" device can indicate which segment crosses over the other when the knot or link is viewed from the direction of the projection. Tait restricted his attention to diagrams with crossings that go alternately over and under as we travel along the knot or link in either direction. A knot with such a diagram is said to be alternating. In "On knots" Tait did not ask whether every knot is alternating. He believed that the answer was no, but he had no means to prove it. Examples of nonalternating knots were later given by C. Bankwitz in 1930 and by Kurt Reidemeister in 1932.

Tait employed a simple code to describe knots. He was apparently unaware that it had been used many years before by Gauss:

If we affix letters to the various crossings, and going continuously round the curve, write down the name of each crossing in the order in which we reach it, we have, as will be

the fact, had written to Tait about it. In his collected works, Klein added a footnote to his paper, stating that the result should follow. 
proved later the means of drawing without ambiguity the projection of the knot.

The problem of classifying knots that can be drawn with a given number of crossings now became one of combinatorics. Unfortunately, some codes cannot be realized. ${ }^{13}$ Those codes that do represent a knot are not unique representations. In order to eliminate duplication, Tait sought tricks that would produce another diagram for a knot from a given one. One trick involved turning over part of the diagram while leaving the rest unchanged. "Flype" was the name that Tait chose for this operation, thereby reviving an old Scottish word meaning roughly to turn outside in, as one might do with a wet glove. Tait made several conjectures that remained unproved for more than a century until deeper methods became available. His conjecture that any two alternating diagrams of the same knot are related by a finite sequence of flypes was finally proved in 1991 by William Menasco and Morwen Thistlethwaite. ${ }^{14}$

In "On knots" Tait mentioned briefly that he had devised a time-saving system using pieces of cardboard "somewhat resembling" John Napier's Radbology. The latter was a system of marked ivory rods with which multiplication and division can be reduced to the simpler operations of addition and subtraction. It was a creation of John Napier, the inventor of logarithms, more than two hundred fifty years earlier. Napier, whose Scottish name admitted a disturbing variety of spellings, often signed himself "Neper." His ivory rods became widely known as "Neper's bones."

\section{Clear Your Coils}

Tait pictured a knot diagram as a rope (with its ends connected) wound on top of an invisible flat surface. Thrust your arm through an empty space in the surface and lift. The result is what Tait meant by a "coil." If all the cords travel in the same direction around your arm, then the coil is "clear." Tait referred to a clear coil as a "perfect plait". In modern parlance, a clear coil is a closed braid.

Of course it might be impossible to clear the coil. A cord might double back and twist about itself, forming a "kink." Or two cords doubling back in opposite directions might pass through each other and "lock.""

\footnotetext{
${ }^{13}$ This difficulty motivated Louis Kauffman in 1991 to develop a theory of virtual knots.

${ }^{14}$ Tait made several conjectures about knots. Another, that a diagram of an alternating link with no reducible crossings has in fact the fewest possible crossings, was proved independently in 1987 by Kauffman, Menasco, and Kunio Murasugi. A reducible crossing is one that can be removed by simply twisting the diagram.

${ }^{15}$ Tait, it seems, almost stumbled onto braid theory, a study introduced by Emil Artin in 1925. What Tait did not seem to
}

\section{Beduped, Befooled, Besotted}

Like Maxwell, Tait was well educated. He astounded friends by reciting from memory lengthy Greek and Latin verse. In "On knots" we see him struggling to find the best phrase to describe a new mathematical phenomenon.

Tait defined the term "order of knottiness" (or simply "knottiness") of any knot to be the smallest number of crossings needed to depict it. For example, a trivial knot, i.e., a planar circle, has knottiness zero. Tait showed that knottiness equal to 1 or 2 is impossible. A trefoil knot has knottiness equal to 3 , since it can be represented by a diagram with 3 crossings. ${ }^{16}$

Knot theorists today use the term "crossing number" instead of "knottiness." Regardless of what word one uses, the quantity is difficult to compute. It is a testimony to the man's courage that Tait introduced a second, much scarier, term, "beknottedness," and promised to explore it in a second paper. By beknottedness Tait meant the smallest number of crossing changes (interchanging over- and underarcs of a diagram) required to change a diagram to that of a trivial knot. "Belinkedness," Tait explained, is defined for links in a similar fashion. Here is the babe entering a dangerous wood.

Beknottedness is frightening for two reasons. First, one must be able to recognize the trivial knot after changing some number of crossings of a diagram. Even today, no efficient algorithm for this recognition problem is known. ${ }^{17}$ Second, in order to obtain a useful quantity, one must find the minimum beknottedness over all diagrams of the knot.

Tait and Maxwell were both "beduped" and "befooled" as they thought about beknottedness. On a piece of paper in a scrapbook that Tait kept, we find an undated note (Figure 3) in which Tait struggles with the concept. On top of the paper we read: "Hitherto measured Bek. [Beknottedness] by least no. of signs to be changed to take all off. Seems unnatural." The words "silver" and "copper" appear below the first pair of diagrams. They refer to imaginary silver and copper coins, which Tait used in various ways as markers. He gives bucolic instructions in "On knots":

realize is that every knot can be put into the form of a closed braid, a theorem proved by J. W. Alexander in 1923.

${ }^{16}$ Tait could not prove that the trefoil knot is different from the planar circle. This fact seemed obvious to him. It would be shown rigorously in 1905 by W. Wirtinger using group theory.

${ }^{17}$ In 1961, W. Haken showed that the problem is at least decidable. His algorithm and later ones require time that is, in the worst cases, exponential in the number of crossings of the knot diagram. 


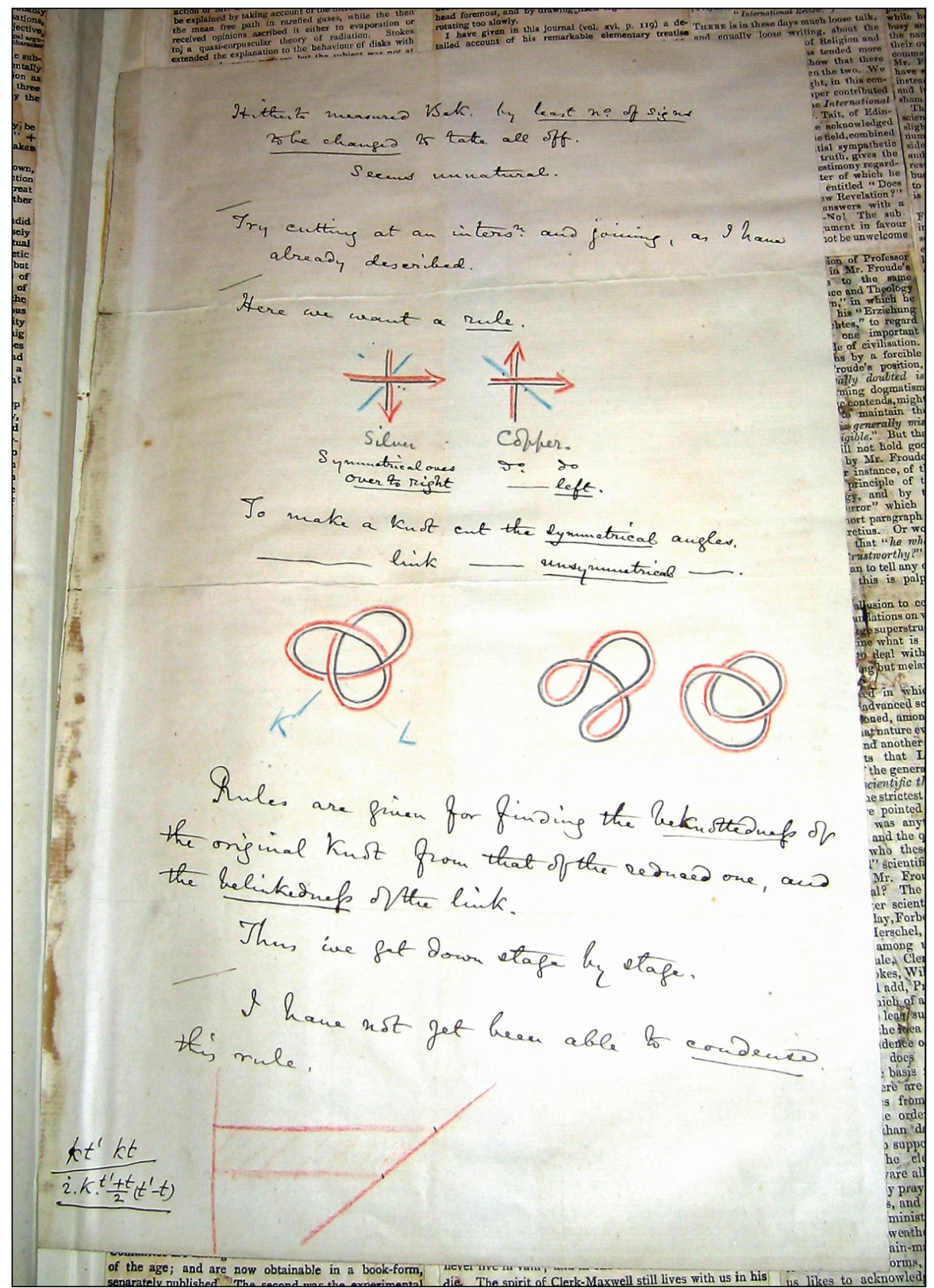

Figure 3. Tait's notes on beknottedness. (Reproduced with permission of James Clerk Maxwell Foundation.) Photo: Susan G. Williams.

Regarding the [knot] projection as a wall dividing the plane into a number of fields, if we walk along the wall and drop a coin into each field as we reach it, each field will get as many coins as it has corners.... To make this process give the distinction between crossing over and cross under, we may suppose the two coins to be of different kinds,--silver and copper for instance.... Or, still more simply, we may dispense altogether with the copper coins, so that going round, we pitch a coin into the field to the right at each crossing over, to the left at each crossing under.
Maxwell will have fun with pitching coins in the last stanza of his poem.

We digress in order to point out a knottheoretical surprise in Tait's note (Figure 3). He has cut a directed knot diagram at a crossing and reconnected the four ends in the two possible ways that respect the direction of the diagram: a "reduced knot" and a link result, each having a diagram with one less crossing than the original. Tait writes: "Rules are given for finding the beknottedness of the original knot from that of the reduced one, and the belinkedness of the link. Thus we get down stage by stage." Tait adds: "I 
have not yet been able to condense this rule."18 In On knots, Part II, Tait discusses beknottedness, and it is likely from what he writes that his notes were written prior to its publication. However, in the later paper he says nothing about the rule he had been seeking.

Had Tait succeeded in condensing his rule, he would have produced the first "skein relation" for knots. Fifty years later, J. W. Alexander would do this. John Conway would introduce the term "skein relation" in 1967, and later developments by Louis Kauffman and Vaughan Jones would make the theory of skein relations one of the most powerful tools of modern knot theory, helping to answer conjectures (such as Tait's Flyping Conjecture) and building bridges between knot theory and mathematical physics.

\section{It's Monstrous, Horrid, Shocking}

Maxwell had a special interest in the Borromean rings, the link that had so perplexed Tait. No two of its rings are linked, yet the configuration cannot be pulled apart. In the language of Tait, the rings were "interlocked" but not "linked." In his Treatise, Maxwell had written about a related but simpler example, two rings with signed or algebraic linking number equal to zero but again impossible to separate, known today as a Whitehead link (Figure 4). Maxwell knew that linking numbers could be computed using an integral formula that Gauss had discovered in 1833 while investigating celestial mechanics. By reinterpreting the integral, Maxwell had realized that, if electric current runs through one ring of the Whitehead link, then no work is done moving a charged particle along the other. Maxwell, an exceptionally able mathematician, sensed a deep idea here. Finer tools than those which he or Tait possessed would be needed to understand it fully.

\section{Little Jacky Horner}

Maxwell returns to children's themes in the final stanza of (Cats) Cradle Song. Tait has been transformed from a reciter of lessons, as in the initial stanza, to a child sitting in a corner, pulling fruit from a pie.

\footnotetext{
${ }^{18}$ One of the referees comments: "Tait had the idea of using an UNORIENTED skein expansion that is exactly the one that sits behind the structure of the Kauffman Bracket state sum model for the Jones polynomial. It was very difficult for researchers (such as myself) to think about unoriented diagrams. The Figure 5 shows that a certain simplicity of approach had been present with Tait, and later lost to others until they found their way back to it!"
}

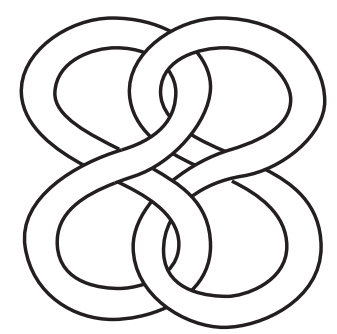

Figure 4. Whitehead link from Maxwell's Treatise.

Little Jack Horner

Sat in the Corner,

Eating a Christmas pie;

He put in his thumb,

And pulled out a plum, And said, 'What a good boy am I.'

The nursery rhyme was already well known in Britain by the eighteenth century. ${ }^{19}$ Lord Byron refers to Jack Horner in his satiric poem Don Juan, Canto XI, published in 1823, a popular work that Maxwell would possibly have read.

It is likely that, in Maxwell's mind, Jack was doing more than playing with his food: he was computing. At the time, most mathematicians hearing the name "Horner" would have immediately thought of Horner's method in algebra. ${ }^{20}$

Jack, sitting in the corner of a knot diagram, would need the silver and copper coins in order to calculate. And by showing us what is proper, as Maxwell promises, the child will lift the fog of our knotful confusion-eventually.

\section{Acknowledgments}

We are grateful to the Simons Foundation for travel support, and the James Clerk Maxwell Foundation, Edinburgh, for making its materials available. We also thank Louis Kauffman, Jozef Przytycki, Susan G. Williams, and the referees for many helpful suggestions.

\section{References}

[1] L. CAMPBELL and W. GARnet, The Life of James Clerk Maxwell, Macmillan and Co., London, 1882.

[2] M. EPPLE, Die Entstehung der Knotentheorie, Friedr. Vieweg \& Sohn Verlagsgesellschaft mbH, Braunschweig/Wiesbaden, 1999.

${ }^{19}$ The rhyme appears in a ballad Namby Pamby, published by William Carey in 1725.

${ }^{20}$ Sir George Gabriel Stokes, a friend of Maxwell, was such a mathematician. In a letter of 1857 to Lady Stokes, Pembroke College, Stokes writes:

I have not forgotten little Jack Horner who sat in a corner, so I comprehend the plum, though nowadays the name Horner is rather associated in my mind with Horner's method of solving numerical equations. 
Mathematics at the National Security Agency

\section{Rise Above the Ordinary}
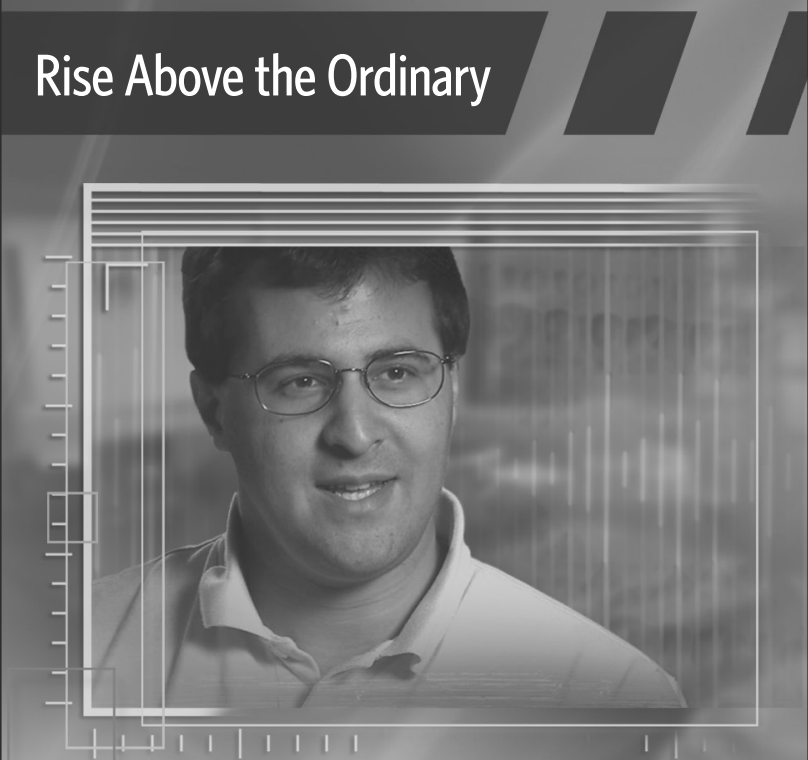

A career at NSA is no ordinary job. It's a profession dedicated to identifying and defending against threats to our nation. It's a dynamic career filled with challenging and highly rewarding work that you can't do anywhere else but NSA.

You, too, can rise above the ordinary. Whether it's producing valuable foreign intelligence or preventing foreign adversaries from accessing sensitive or classified national security information, you can help protect the nation by putting your intelligence to work.

NSA offers a variety of career fields, paid internships, c0-op and scholarship opportunities.

Learn more about NSA and how your career can make a difference for us all.

\section{KNOWINGMATTERS}

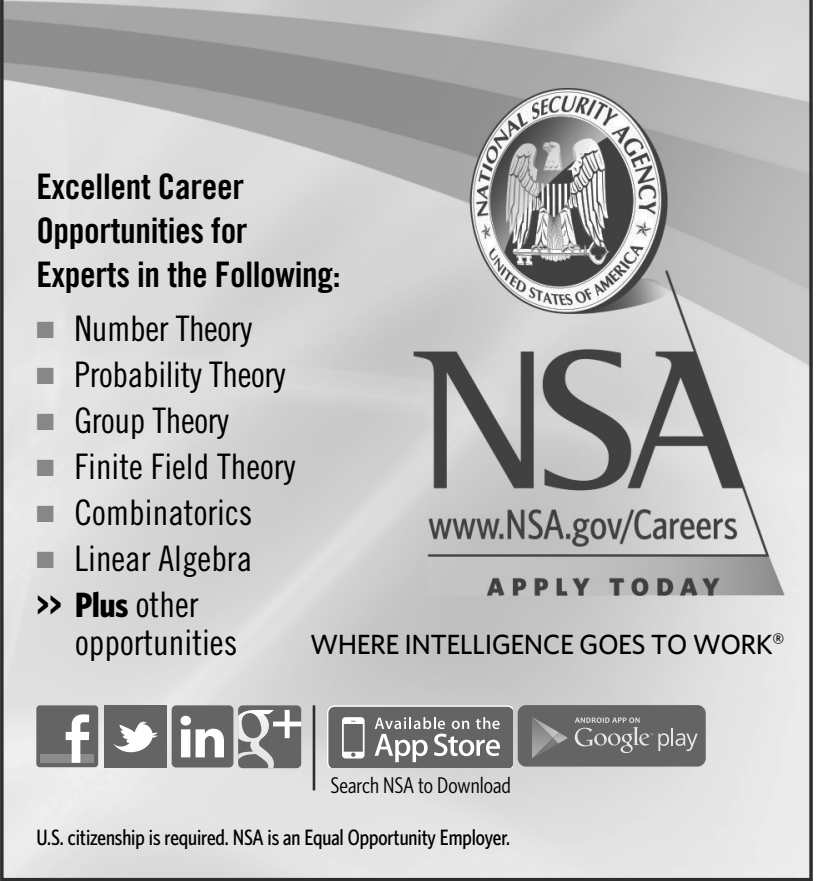

[3] H. von HeLmholtz, Ueber Integrale der hydrodynamischen Gleichungen welche der Wirbelbewegung entsprechen, Journal für reine und angewandte Mathematik 55 (1858), 25-55.

[4] C. G. KNotT, Life and Scientific Work of P. G. Tait, Cambridge University Press, 1911.

[5] P. M. HARMAN (ed.), The Scientific Letters and Papers of James Clerk Maxwell, 3 volumes, Cambridge University Press, 1990, 1995, 2002.

[6] H. vON HeLmHOLTz, On the integrals of hydrodynamic equations to which vortex motion conforms, Crelle's J. f. reine u. angew. Mathematik, LV, 1858 (translated in Phil. Mag. (4), XXXIII).

[7] L. C. KAUFFMAN, New invariants in the theory of knots, Asterisque 163-164 (1988), 137-219; and Amer. Math. Monthly 95, no. 3 (March 1988), 195-242.

[8] F. C. KLEIN, Ueber den Zusammenhang der Flächen, Math. Annalen, ix, 1876, 476-482.

[9] J. B. LisTING, Vorstudien zur Topologie, Böttinger Studien 2 (1847), 811-875; published separately, Göttingen: Vandenhoeck und Ruprecht, 1848.

[10] , Der Census räumlicher Complexe, Abhandlungen der Königlichen Gesellschaft der Wissenschaften zu Göttingen, Math. Classe 10 (1861), 97-182; published separately, Göttingen: Diederichsche Buchhandlung, 1862.

[11] J. C. MAXWELL, A Treatise on Electricity and Magnetism, 2 vols., Oxford: Clarendon Press, 1873.

[12] J. H. PRZYTYCKI, The Trieste look at knot theory, L. H. Kauffman et al. (eds.), Introductory Lectures on Knot Theory, Series on Knots and Everything, 46, World Scientific, pp. 407-441, 2012.

[13] D. S. SILVER, "Perhaps I might explain this": The toys and humor of James Clerk Maxwell, in Textualities, Science and Intuition issue, Edinburgh, 2007.

[14] , Knot theory's odd origins, American Scientist 94, no. 2 (2006), 158-165.

[15] _ , The last poem of James Clerk Maxwell, Notices of the American Mathematical Society 55 (2008), 12661270.

[16] O. Simony, Gemeinfassliche, leicht controlirbare Lösung der Aufgabe: "In ein ringförmig geschlossenes Band einen Knoten zu machen" und verwandter merk würdiger Probleme, Verlag von Gerold \& Comp., Wien, 1881.

[17] B. STEWART and P. G. TAIT, The Unseen Universe or Physical Speculations on a Future State, Macmillan and Co., London, 1875.

[18] G. G. STOKes, Memoir and Scientific Correspondence, Volume L, Cambridge University Press, Cambridge, 1907.

[19] P. G. TAIT, Lectures on Some Recent Advances in the Physical Sciences, Macmillan and Co., London, 1876.

[20] _ On knots, Transactions of the Royal Society of Edinburgh 28 (1877), 145-190.

[21] _ On knots, Part II, Transactions of the Royal Society of Edinburgh 32 (1884), 327-342.

[22] _ On knots, Part III, Transactions of the Royal Society of Edinburgh 32 (1885), 335-347.

[23] _ , On the teaching of natural philosophy, Contemporary Review, January 1878.

[24] _ , Notice of J. B. Listing, Nature 27 (1883), 316317.

[25] _ Listing's Topologie, Philosophical Magazine, January 1884 